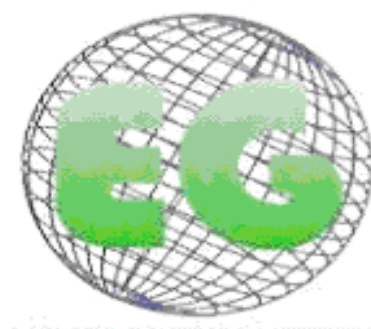

ISSN 1695.6141 $N^{\circ} 26$

\title{
Relaciones sociales de género de las matronas en una comarca rural de España
}

Gender-based social relationships of midwives in a rural district in Spain

\author{
*Linares Abad, M., **Moral Gutiérrez, I., "Alvarez Nieto, C., *Grande Gascón, ML., \\ ${ }^{*}$ Pancorbo Hidalgo, PL.
}

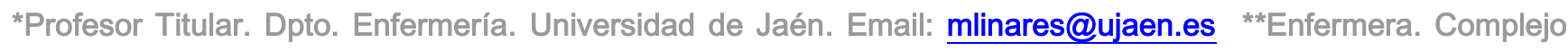
Hospitalario de Jaén.
\end{abstract}

Palabras clave: matrona; cuidado; relaciones sociales de género; estudios de mujeres

Keywords: midwife; history; gender-based relationships; women' s studies

\section{RESUMEN}

Objetivos: Son muchas las investigaciones que cada vez dan más importancia al Estudio de las Mujeres teniendo en cuenta el contexto de las relaciones sociales de género. Desde este marco teórico nos hemos acercado a las matronas que realizaron su trabajo en una comarca rural situada en el sur de España (Sierra Mágina, Jaén) para conocer las relaciones sociales que configuraron en torno a su profesión.

Diseño. Investigación cualitativa etnográfica mediante entrevistas individuales y grupos de discusión.

Lugar: Comarca rural de Sierra Mágina (Jaén, España).

Participantes: 9 matronas y 11 familiares directos de matronas de esta comarca que ejercieron la profesión en la segunda mitad del siglo XX. 16 grupos de discusión con mujeres de los pueblos de la comarca con experiencias de haber sido atendidas por matronas.

Hallazgos: Hemos descrito y analizado a través de informantes y matronas, las relaciones que éstas últimas establecieron con la comunidad y otros profesionales de la salud en los municipios donde trabajaron. Con la comunidad unas matronas establecieron relaciones jerárquicas y otras relaciones igualitarias. Con practicantes y ayudantes técnicos sanitarios (ATS) configuraron relaciones basadas en intereses laborales; con médicos las relaciones fueron complejas y estrechas dada la interdependencia a la que estaban sometidos, y con parteras, en general, las relaciones fueron distantes aunque a veces hubo relaciones de conveniencia.

Conclusiones: El estudio de las relaciones diarias entre vecinos y profesionales de la salud, y el estudio del impacto de la labor realizada por las matronas nos acerca a su realidad, a su estatus, y a las relaciones de poder. 


\section{ABSTRACT}

Objective: Increasingly research is giving more importance to Women's Studies taking into account the context of gender-based social relationships. From this theoretical framework we have focused on the work carried out by midwives in a rural district in southern Spain (Sierra Mágina, Jaén), in order to learn about the social relationships established around their profession.

Design: Ethnographic qualitative research using personal interviews and discussion groups.

Setting: Rural district of Sierra Mágina (Jaén, Spain).

Participants: 9 midwives and 11 close relatives of midwives from this rural district who worked there during the second half of the twentieth century. Also, there were 16 discussion groups with women from towns in the district who had been assisted by midwives.

Findings: We have described and analysed discussions with both informants and midwives themselves, the relationships the midwives established in the community and with other health professionals in the districts where they worked.

Conclusions: The study of the daily interaction between neighbours and healthcare professionals, and the study of the impact caused by the work performed by midwives brings us closer to their reality, their status, and empowerment.

\section{INTRODUCCIÓN}

Los estudios sobre mujeres y género abren nuevas líneas de investigación donde emerge con fuerza el estudio de la llamada gente corriente o historia desde abajo ${ }^{(1)}$; no sólo es importante el estudio de grandes mujeres. Además se está prestando mucha atención al estudio de las mujeres en las profesiones sanitarias. Investigaciones en este campo apuntan al estudio de las mujeres en el marco de las relaciones sociales de género y dentro del contexto de las estructuras económicas, culturales, políticas y religiosas que articulan la sociedad contemporánea ${ }^{(2)}$. En lo que a profesiones sanitarias y mujeres se refiere, analizar y construir la identidad de las matronas como colectivo es una línea de investigación interesante y novedosa apuntada por las expertas en este tema. La evolución histórica de las matronas y sus relaciones con las enfermeras a lo largo del siglo XX ha sido estudiada por varios autores, tanto en España ${ }^{(3,4,5)}$ como en otros países ${ }^{(6)}$.

De esta forma, nuestro propósito es contribuir a la elaboración de una historia más universal preocupada por la experiencia de la participación no sólo de hombres, sino también de mujeres que han contribuido socialmente a la construcción de esta sociedad. Reconocer y dar autoridad mediante la investigación a las experiencias sanitarias de las mujeres en el pasado es una forma de reconocer y dar autoridad en el presente a todas las mujeres trabajadoras y en especial a las del ámbito de la salud ${ }^{(7)}$.

Bajo este marco teórico el objetivo de este estudio es describir y analizar las relaciones sociales de género que las matronas de una comarca rural del sur de España (Sierra Mágina) establecieron en los municipios donde ejercieron su profesión con sus habitantes, con las parteras tradicionales y con otros profesionales sanitarios, como médicos, practicantes y ATS. (Los practicantes y los ATS fueron profesionales de enfermería, de formación muy práctica y casi siempre hombres, que ejercieron en España durante el siglo $X X)$. 


\section{MÉTODOS}

\section{Diseño}

Se trata de una etnografía local de carácter retrospectivo, descriptivo y de análisis de contenidos. Los datos fueron recogidos en varios períodos entre febrero de 2004 y noviembre de 2006.

\section{Entorno}

El ámbito de estudio es una comarca rural situada en el sur de España (en la provincia de Jaén). Comprende un total de 16 pueblos con una población entre 531 y 12115 habitantes. Hasta el año 1950 el crecimiento de población en la comarca fue ascendente, a partir de este año se ve bruscamente invertido debido a los procesos migratorios hacia el norte de España. Las causas de esta transformación radicaron en la grave crisis económica y la dureza de las condiciones de vida posteriores a la Guerra Civil Española, así como al aislamiento geográfico y social de la comarca que se mantuvo al margen del desarrollo industrial de los años 50 y 60 provocando el éxodo de los pueblos por parte de los habitantes más jóvenes de la comarca, dando como resultado el envejecimiento de la población y un descenso demográfico que en determinados momentos alcanza hasta el $50 \%$ (Asociación para el desarrollo rural de Sierra Mágina) . El estudio se refiere a las relaciones establecidas durante la segunda mitad del siglo XX.

\section{Participantes}

Se han seleccionado dos grupos de participantes para esta investigación: a) mujeres de esta comarca que tuvieron experiencias de embarazo y parto durante el periodo estudiado; y b) matronas y familiares directos de ellas que ejercieron su profesión en esta comarca.

\section{Mujeres experimentadas}

Con el propósito de recoger datos mediante grupos de discusión nos pusimos en contacto con las presidentas de las Asociaciones de Mujeres de los pueblos de la comarca de Sierra Mágina. Le explicamos telefónicamente los objetivos de nuestro estudio y les comunicamos que queríamos seleccionar entre 6 y 8 informantes mujeres que cumplieran los siguientes criterios;

-Que tuvieran memoria histórica.

-Nacidas y residentes en los municipios estudiados.

-Edad superior a 60 años.

-Madres o con experiencia directa con la asistencia a la maternidad.

Hemos recogido información para esta investigación de 16 grupos formados en los pueblos de la comarca (Albanchez de Mágina, Bélmez de la Moraleda, Cambil-Arbuniel, Mancha Real, La Guardia de Jaén, Jimena, Noalejo-Hoya del Salobral, Pegalajar, Huelma-Solera, Larva, Torres, Los Cárcheles, Cabra del Santo Cristo, Jódar, Campillo de Arenas y el último Bedmar-Garcíez).

\section{Matronas y familiares}

Para identificar a las matronas que trabajaron en la Comarca de Sierra Mágina durante el siglo XX, se partió del único libro de registro de títulos de matronas archivado en el Colegio 
Oficial de Enfermería de Jaén. De las 201 matronas inscritas en este libro que trabajaron en la provincia de Jaén, 29 lo hicieron en la comarca de Mágina. Las mujeres experimentadas de la comarca también nos aportaron datos sobre familiares directos de las matronas de la Comarca, direcciones postales y números de teléfono de las matronas objeto de estudio. Esto permitió entrevistar a nueve matronas (Tabla 1) que viven en la actualidad y a once familiares de matronas ( 3 hijas, 2 hijos, 1 hermana y 1 sobrina).

Tabla 1. Datos sociodemográficos y laborales de las matronas entrevistadas

\begin{tabular}{|c|c|c|c|c|c|c|c|c|c|}
\hline $\begin{array}{l}\text { Iniciales de } \\
\text { las matronas }\end{array}$ & A.A.P. & J.C.L. & J.D.M. & M.G.M. & F.G.C. & I.L.R. & I.M.L. & E.R.H. & M.S.S. \\
\hline $\begin{array}{l}\text { Lugar de } \\
\text { nacimiento }\end{array}$ & $\begin{array}{l}\text { Fuerte del } \\
\text { Rey (Jaén) }\end{array}$ & $\begin{array}{l}\text { Arjona } \\
\text { (Jaén) }\end{array}$ & $\begin{array}{l}\text { Bedmar } \\
\text { (Jaén) }\end{array}$ & $\begin{array}{l}\text { Jódar } \\
\text { (Jaén) }\end{array}$ & $\begin{array}{l}\text { lbros } \\
\text { (Jaén) }\end{array}$ & $\begin{array}{l}\text { Cabra } \\
\text { Santo } \\
\text { Cristo } \\
\text { (Jaén) }\end{array}$ & $\begin{array}{l}\text { Úbeda } \\
\text { (Jaén) }\end{array}$ & $\begin{array}{l}\text { Fuerte } \\
\text { del Rey }\end{array}$ & $\begin{array}{l}\text { Valdepeñas } \\
\text { (Jaén) }\end{array}$ \\
\hline $\begin{array}{l}\text { Edad actual } \\
\text { Estado civil } \\
\text { Número de } \\
\text { hijos/as }\end{array}$ & $\begin{array}{l}78 \text { años } \\
\text { Casada } \\
3\end{array}$ & $\begin{array}{l}77 \text { años } \\
\text { Casada } \\
3\end{array}$ & $\begin{array}{l}76 \text { años } \\
\text { Casada } \\
2\end{array}$ & $\begin{array}{l}92 \text { años } \\
\text { Soltera }\end{array}$ & $\begin{array}{l}94 \text { años } \\
\text { casada } \\
2\end{array}$ & $\begin{array}{l}70 \text { años } \\
\text { casada } \\
6\end{array}$ & $\begin{array}{l}79 \text { años } \\
\text { casada } \\
3\end{array}$ & $\begin{array}{l}76 \text { años } \\
\text { casada } \\
2\end{array}$ & $\begin{array}{l}81 \text { años } \\
\text { casada } \\
4\end{array}$ \\
\hline $\begin{array}{l}\text { Universidad } \\
\text { que expide } \\
\text { el título }\end{array}$ & Sevilla & Granada & $\begin{array}{l}\text { Central } \\
\text { de } \\
\text { Madrid }\end{array}$ & Granada & Granada & Granada & $\begin{array}{l}\text { Central } \\
\text { de } \\
\text { Madrid }\end{array}$ & Cádiz & Sevilla \\
\hline $\begin{array}{l}\text { Pueblos } \\
\text { donde } \\
\text { trabajó }\end{array}$ & $\begin{array}{l}\text { Carchelejo } \\
\text { Noalejo }\end{array}$ & Noalejo & Pegalajar & $\begin{array}{l}\text { Garcíez } \\
\text { (Bedmar) }\end{array}$ & Huelma & $\begin{array}{l}\text { Huelma } \\
\text { Cabra } \\
\text { Santo } \\
\text { Cristo }\end{array}$ & Jódar & $\begin{array}{l}\text { La } \\
\text { Guardia }\end{array}$ & $\begin{array}{l}\text { La } \\
\text { Guardia }\end{array}$ \\
\hline $\begin{array}{l}\text { Tiempo de } \\
\text { ejercicio } \\
\text { profesional }\end{array}$ & $\begin{array}{l}6 \text { años } \\
(1951- \\
1956\end{array}$ & $\begin{array}{l}3 \text { años } \\
1950- \\
1951\end{array}$ & $\begin{array}{l}2 \text { años } \\
1960- \\
1962\end{array}$ & $\begin{array}{l}4 \text { años } \\
1936- \\
1939\end{array}$ & $\begin{array}{l}15 \text { años } \\
1948- \\
1962\end{array}$ & $\begin{array}{l}8 \text { años } \\
1960- \\
1967\end{array}$ & $\begin{array}{l}\text { 25años } \\
1951- \\
1975\end{array}$ & $\begin{array}{l}5 \text { años } \\
1951- \\
1955\end{array}$ & $\begin{array}{l}37 \text { años } \\
1955-1988\end{array}$ \\
\hline
\end{tabular}

\section{Obtención de datos}

Los instrumentos utilizados para la recogida de información fueron los propios de la investigación etnográfica, grupos de discusión y entrevista semiestructurada.

\section{Grupos de discusión}

El grupo de discusión es una técnica de recogida de datos que aporta conocimientos sobre actitudes, percepciones y opiniones de los participantes, se obtiene la información mediante preguntas abiertas a los informantes. Los grupos se construyeron siguiente las recomendaciones de la literatura ${ }^{(8)}$. Los datos relacionados con las opiniones y percepciones se enriquecen por medio de la interacción de las personas, esto es debido a que la participación individual se puede mejorar en un escenario grupal ${ }^{(9)}$. Las sesiones de los grupos de discusión se hicieron durante la tarde (excepto en dos casos), aprovechando la mayor disponibilidad de tiempo de las personas entrevistadas. El lugar de realización fue en torno a una mesa en las sedes de las Asociaciones de Mujeres de cada pueblo, excepto dos, una en la sala de juntas del Ayuntamiento y otra en la casa de la presidenta de la asociación.

Antes de comenzar la dinámica de grupo propiamente dicha, la presidenta de las respectivas asociaciones de mujeres presentó al investigador, que actuó como moderador. Para minimizar el fenómeno del forastero, se explicó detalladamente cuál era la finalidad de la reunión y la confidencialidad con la que se iban a tratar los datos recogidos. Se estableció como norma, antes de iniciar las sesiones, que se respetara el turno de intervención de las 
participantes, para evitar errores y solapamientos en la posterior transcripción. Al principio, cada vez que una participante intervenía se presentaba con su nombre de pila hasta que intentamos memorizar el nombre de la persona que intervenía. No hemos percibido extrañamiento ni conducta cohibida en las participantes, aunque en algunas ocasiones hemos tenido que alentar la participación de las personas más introvertidas y reconducir a las participantes extrovertidas y más dispuestas a participar, colocándonos estratégicamente en el grupo al lado de las primeras. La duración de las sesiones osciló entre 2 y 4 horas. El rango de edad de las mujeres estaba en 65 años de la más joven y 94 años de la mayor. Todos los grupos de discusión se desarrollaron

\section{Entrevistas semiestructuradas}

La entrevista en profundidad pretende la comprensión de los diferentes puntos de vista que los informantes tienen de sus experiencias de vida. Una de las aportaciones fundamentales que conlleva la utilización de esta estrategia es que nos da información que se construye a caballo entre el discurso y la conducta; de hecho la información que recogemos a través de la entrevista es elaborada por el propio informante a partir de su experiencia con el tema investigado y condicionado por la persona que realiza la entrevista y la situación de entrevista ${ }^{(10)}$.

Todas las entrevistas fueron realizadas en el domicilio de los informantes, excepto dos (en una residencia de ancianos y en casa de un familiar). Se efectuó un contacto telefonico previo, presentando al investigador y los objetivos del estudio y solicitando su participación. En ese primer contacto fijamos una fecha y lugar para realizar la entrevista. En el día de la entrevista insistimos, al comienzo, sobre los objetivos pretendidos, les comentamos la necesidad de utilizar la grabadora de audio para registrar la información y poder así realizar una transcripción más fiel de los contenidos. Por el respeto hacia los participantes y los problemas éticos que plantean las investigaciones cualitativas ${ }^{(11)}$, ofrecimos la confidencialidad a los informantes; no fue necesaria en el caso de las matronas y los familiares, se sintieron orgullosas y muy agradecidos de que estuviéramos inmersos en una investigación que reconocía y rescataba de la memoria a ellas mismas, a sus madres, hermanas o tía en cada caso. Para las informantes no emparentadas seguimos la misma tónica que para los grupos de discusión en cuestiones de confidencialidad. En todos los casos procuramos crear un clima distendido para que los entrevistados se expresaran con total naturalidad. La duración de las entrevistas tuvo un rango entre una hora y treinta minutos de la más corta a tres horas y treinta minutos la de mayor duración.

\section{Análisis}

El proceso de análisis elegido para abordar los datos ha sido el análisis de contenido y el análisis del discurso. El tratamiento o procesamiento de los datos lo hicimos siguiendo el modelo de Huberman y Miles ${ }^{(12)}$ : reducción de los datos, presentación de los datos y por último la extracción y verificación de conclusiones - fase esta última donde recae la interpretación de los datos extrayéndose su significado. Como ayuda para el análisis se utilizó el programa Nudist Vivo.

\section{Aspectos éticos}

Para todas las participantes tuvimos en cuenta los aspectos éticos de toda investigación, ofreciendo a todas las entrevistadas la confidencialidad de los datos, igualmente pedimos permisos para utilizar grabadora al objeto de hacer una trascripción más fiel de la información recogida y también nos permitieron tomar fotografías. 


\section{Hallazgos}

\section{Relaciones con la Comunidad}

Las relaciones que establecieron con la gente dependieron del tiempo que estuvieron ejerciendo su profesión, de la coincidencia entre el lugar donde nacieron y ejercieron y sobre todo del carácter particular de cada matrona. Tuvieron enfoques diferentes en la conceptualización de las relaciones personales: las hay que establecieron una relación jerárquica con los vecinos a semejanza de la que ellas traían de su formación académica; otras se integraron como parte de la comunidad en una relación más igualitaria. Las mujeres de los pueblos sirvieron para reforzar el estatus de las matronas, eran las matronas la referencia para la asistencia del parto normal aunque en algunos pueblos tardaron en adaptarse y hubo más resistencia a la nueva figura profesional. Las matronas fueron respetadas y se movieron entre relaciones maternalistas y relaciones profesionales. $\mathrm{El}$ tratamiento que siempre les dispensaron fue el de doña, aún cuando muchas llegaron a estos pueblos con la carrera recién terminada y a una edad muy joven. Bien es verdad que hemos advertido una relación entre la utilización del nombre en diminutivo o de forma familiar, (precedido del tratamiento de doña), y la edad joven de la matrona; doña Paquita, doña Encarnita, doña Manolita, doña Isabelita por poner algunos ejemplos. Esto lo interpretamos como una muestra de mantener el estatus y el respeto de la matrona y a la vez querer establecer una relación de cercanía y familiaridad con ella.

Cuando hemos preguntado por invitaciones a actos sociales oficiales o institucionales nos hemos encontrado con que el estatus de alguna de las matronas era reforzado con una palma cuando se iniciaban los desfiles procesionales de la Semana Santa, en otras ocasiones eran obsequiadas con pases para espectáculos como corridas de toros o bailes en las fiestas patronales:

En el Domingo de Ramos nos regalaban la Palma. No era corriente invitar a mujeres. En fiestas patronales y fiestas religiosas alguna copa de vino a médicos, practicantes $y$ cabo de la Guardia Civil (E. R. H., matrona en La Guardia).

Fuera de estas particularidades, las matronas en Sierra Mágina no han participado en actos institucionales como nos han dicho nuestras informantes:

Las matronas bastante tenían con estar pendientes de nosotras las 24 horas del día y luego atender sus casas. Antes no se celebraban tantas cosas como ahora. (Informante de Bedmar)

Las matronas han sido también invitadas de forma irregular al acto social y religioso del bautismo del recién nacido como muestra de agradecimiento por la asistencia a la madre en el momento del parto. En la época que hemos estudiado esta invitación no tenía la finalidad de tiempos anteriores, que la matrona llevara al recién nacido a la pila bautismal como era costumbre y tradición (13). Tampoco fueron grandes celebraciones algunas consistían en bautizar al recién nacido sin más celebración que tomar un chocolate en familia o arrojar unos cuantos caramelos y peladillas a la chavalería que lo demandaba en la calle detrás de la madrina y el padrino. Las celebraciones se realizaban en función de la economía familiar. La presencia de la matrona en el bautizo de recién nacidos de la realeza en épocas anteriores fue un signo de prestigio social para la matrona. A mediados del siglo XX era un acto que se iba perdiendo aunque las familias adineradas lo hacían con más frecuencia, entendemos que por economía y por acercarse a esas reminiscencias de grandeza del pasado: 
Normalmente te invitaban las familias que más recursos tenían. (I. M. L., matrona en Jódar).

Sí, era frecuente que participaras en el bautizo, una forma más de agradecer el trabajo que realizabas con ellas. (I. L. R., matrona en Cabra del Santo Cristo).

Yo le dije a don Paulino "que no sea esto cosa de que los otros quieran hacer igual, yo le hago esto por una deferencia pero que no me gusta llevar a los niños a la iglesia porque no". (F. G. C., matrona en Huelma).

Es que no se celebraba nada como ahora, que íbamos con el crío y lo acristianábamos y ya está. No era costumbre, a donde le decían a la matrona que fuera era en las casas grandes, en la de los ricos, le decían que fuera a la iglesia y al bautizo, porque es que yo he ido con ella, iba la partera. (Informante de Bedmar).

\section{Relaciones con practicantes-ATS}

La relación que mantuvieron con practicantes y ATS (enfermeros masculinos) fue a la vez una relación de intereses profesionales y de reciprocidad en la ayuda laboral. Las matronas entrevistadas o sus familiares nos han hablado siempre de una relación cordial entre matronas y practicantes-ATS en esta Comarca estudiada, solo en uno de los pueblos parece que hubo algunas fricciones relacionadas con temas económicos. La forma de ausentarse del lugar de trabajo la mayoría de las veces pasaba por advertir al médico y al ayuntamiento de la ausencia y pactar con ellos la cobertura de los servicios que quedaban descubiertos. Aún cuando los practicantes desde principios del siglo $X X$ recibían en su periodo de formación enseñanzas para la asistencia al parto, y a algunos le acumularon la plaza de matrona por vacante en los pueblos donde ejercieron, no nos han informado de conflictos por injerencias de estos en el trabajo de las matronas. Los practicantes y ATS supieron retirarse de la esfera del nacimiento a no ser que por necesidad de la titular fueran requeridos para prestar ayuda. Esto creemos que es debido por un lado al reconocimiento de los practicantes hacia la profesionalidad de las matronas y a su parcela de actuación y por otro al pudor y resistencia de las mujeres a ser tratadas en temas de embarazo y parto por hombres. La concepción de épocas anteriores de que el parto es una cuestión de mujeres y entre mujeres ha estado y está aún presente en el imaginario colectivo de las mujeres de los pueblos estudiados.

A continuación presentamos testimonios de mujeres y matronas que refuerzan esta idea que defendemos:

Cuando el practicante cayó enfermo, para que el hombre siguiera cobrando, le hacíamos el trabajo y él cobraba su sueldo. (E. R. H., matrona en La Guardia).

Ellos me hacían a mí favores, las inyecciones a la familia, yo sus partos, en fin éramos uña y carne todos. A mi me decían muchas veces las mujeres "iiAy!! doña Paquita, ¿por qué no viene a ponerme esta caja de inyecciones, que me da vergüenza que el practicante me vea el culo?". "Mire usted si el practicante, ese alto, el no mira el culo", yo le explicaba todo, "el culo no es para ellos nada, si el culo para nosotros es un órgano, que da Dios, uno para salir el feto, otro para que le pinchen, ni el médico, ni el practicante, ni yo nos fijamos en su culo de usted, llámelo usted". Los practicantes locos conmigo, don Antonio, ¿conoce a don Antonio?, pobrecillo, don Francisco se fue a Granada. (F. G. C., matrona en Huelma). 


\section{Relaciones con médicos}

Con los médicos las relaciones fueron más complejas y estrechas, pero en general las matronas establecieron buenas relaciones con los médicos de la comarca de Sierra Mágina dada la interdependencia a la que estaban sometidos. A matronas y médicos les interesaba trabajar con profesionales competentes, cuanto más competente fuera la matrona menos avisos recibía el médico y sobre todo a horas intempestivas. Como en todas las profesiones cuando el equipo forma una piña y se llevan bien son menos vulnerables a los conflictos por posibles negligencias y se prestan a la ayuda mutua en el trabajo. No hemos percibido una relación jerárquica entre estos profesionales en este ámbito de estudio y como hemos dicho anteriormente las matronas de esta comarca forjaron su espacio de trabajo a base de una práctica diaria en condiciones muchas veces adversas y sabiéndose que eran las profesionales que tenían una formación más amplia y específica sobre cuestiones relacionadas con el nacimiento. Con todo, la estructura social y sanitaria del sistema ponía a la matrona a las órdenes del médico.

Los médicos de Sierra Mágina han dejado a las matronas la dirección de los partos, dando como buenas las decisiones que tomaban acerca de las intervenciones que debían realizar: Me decía "lo que tú digas y propongas". (E. R. H., matrona en La Guardia).

Las relaciones con los médicos guardaron también su matiz de complicidad y picaresca, de ellos dependía muchas veces la mayor o menor administración de inyectables y los actos médicos a realizar en una época donde todavía las diarreas infantiles y demás trastornos alimentarios necesitaban de sueroterapia por doquier. En alguna ocasión han querido enjuagar malas actuaciones solicitando a la matrona ser requeridos cuando realmente no había necesidad de su intervención, pero como decimos, esto entraba en el juego de la complicidad corporativa sanitaria.

En general, las relaciones con el colectivo médico han sido percibidas por informantes, familiares y matronas como muy buenas, prueba de ello son algunos de los testimonios que hemos recogido:

Me llevaba con ellos extraordinariamente, ya ve, don Ebelio fue el primero que era de Granada, todos, todos, todos, don Paulino, eran tres uno de ellos particular. Muchas veces decía yo "mire usted esto tiene que venir el médico para ver que opina él, yo también tenía que ver con él. Yo decía "para que lo voy a llamar si esto... Yo he ido con la cosa de que el médico lo supiera. (F. G. C., matrona en Huelma).

Trabajábamos en común, si había un parto distócico pues iba el médico, le decía a la familia que avisara al médico. El médico iba. El primer médico eran dos hermanos los "Siles", luego vino Don Pedro. D.. (M. S. S., matrona en La Guardia).

\section{Relaciones con parteras}

Las relaciones que mantuvieron con las parteras tradicionales o mujeres aficionadas, como también se les llama en esta Comarca, fueron más variables y menos homogéneas que las que establecieron con los profesionales titulados de la salud. Nos ha sorprendido la cantidad de parteras que se dedicaron de forma altruista a la asistencia de las mujeres de su comunidad en tanto en cuanto las matronas tituladas fueron ocupando sus plazas. Fueron mujeres que no pidieron pagos por su trabajo, excepto una partera de Bedmar con autorización del médico para asistir partos, recibían el pago por sus servicios en forma de regalos, productos que cubrían sus necesidades más básicas, desde un mandil o unas 
alpargatas hasta un puñado de garbanzos, pasando por unos trozos de jabón o media alcuza de aceite, pero nunca pedidos por ellas. Era una economía de subsistencia para unas familias donde los ingresos de los hombres no eran suficientes.

Nos consta, por los testimonios recogidos, que hubo un tiempo en el que el trabajo de mujeres aficionadas y matronas se mantuvo simultáneamente, estaban ahí y no se notaban, estaban dispuestas cuando se les necesitaba. A las mujeres de esta comarca les costó tiempo desprenderse de la labor realizada por quienes eran sus vecinas o familiares para dejar ese momento tan íntimo del nacimiento en manos de matronas, que la mayoría de las veces, venían de otros lugares de procedencia. El parto seguía en la esfera de lo femenino pero era el comienzo de un movimiento de alejamiento del proceso de nacimiento desde la esfera de lo privado hacia lo público, desde el concepto de normalidad y espontaneidad al concepto del parto como proceso tecnificado considerado como una enfermedad ${ }^{(14)}$.

Las matronas estudiadas tuvieron que conjugar respeto, jerarquía, acercamiento e integración para ser parte de la comunidad y a la vez estar por encima de la comunidad, para ser vecinas y a la vez profesionales, para que su trabajo poco a poco fuera aceptado. A pesar de todo, esta transición no la hemos percibido traumática a través de los testimonios recogidos. No olvidemos que el discurso higienista de mitad de siglo quería erradicar o eliminar las prácticas basadas en la fuerza de la costumbre sin fundamento científico ${ }^{(15)}$, a pesar de que desde los Consejos Nacionales de Matronas se propugnaba a los cuatro vientos la lucha contra las intrusas. Las parteras de la comarca supieron difuminarse de la esfera del parto sin causar disturbios, sin que las mujeres de parto notaran que llegaba el fin de una figura tan importante como controvertida a lo largo de la historia. Cuando las matronas ya ejercían como tales, las parteras eran necesitadas aún por quienes no tenían cobertura sanitaria o no tenían dinero para pagar los horarios de una titulada a pesar del carácter humanitario que hemos presentado de las matronas. Algunas mujeres se resistían a parir con una extraña cuando los partos anteriores habían tenido un final feliz con las parteras tradicionales mujeres del pueblo. Esta resistencia al cambio de gestoras del parto lo percibieron las matronas y algunas le llamaron proceso de adaptación de las mujeres del pueblo a las matronas, pero ellas tuvieron también que adaptarse al medio donde trabajaron.

Fueron también, aunque no lo han reconocido las matronas abiertamente en el discurso, relaciones de conveniencia y a veces de necesidad, en unas ocasiones la matrona necesitaba ausentarse del pueblo y las parteras descargaban de trabajo a médicos y practicantes que debían suplirla. Aunque había reglamentación que regulaba que la matrona que se ausentara por enfermedad o por asuntos personales tenía que proponer una suplente para que la cubriera, la realidad fue que se ausentaban por un día o dos para ir a la capital por diversos motivos sin que otra matrona la sustituyera, unas veces para ver a sus hijos e hijas que los tenían en colegios de la capital, otras para asistir a cualquier tipo de acto o arreglo de documentación. Hicieron, no todas, caso omiso en algunas ocasiones, sobre todo cuando se ocupaban de mujeres que sabían que recurrían a ellas por la escasez de recursos o se identificaban como familiares directos:

Cuando yo venía a Jaén ella aprovechaba y alguna ya que no querían porque subí la cuota y ya no eran los doce duros, que era ya más dinero, pues las pobres por ahorrarse la llamaban a ella, la que tenía un poquito de dinero y de eso no las llamaban, eran más bien pobrecillas mujeres. (F. G. C., Matrona en Huelma).

Cuando doña Paz se iba de viaje se lo decía a la otra, a Juana "la candileja". Cuando doña Paz estaba aquí si se tenía que ir a algún sitio también, pues se quedaba el médico o el practicante. Cuando doña Paz estuvo de viaje yo estaba mala y dije "Dios 
quiera que esté aquí cuando sea mi parto", nos daba más vergüenza del practicante. (Informante de Torres).

También se dieron situaciones de partos simultáneos y la matrona ordenaba a las vecinas que avisaran a estas mujeres parteras para que se ocuparan, generalmente, del parto que presentaba menos complicación:

La mujer bajó dos o tres veces al día pero aquella noche se puso otra mujer de parto la de "piernas" que está casada con el jabonero, ¿Te acuerdas tu de ella? y le venía la chiquilla muerta y entonces subió ya que estaba el parto y le dijo doña María a mi madre y a mi suegra "Que asistas a tu hermana porque yo no puedo dejar sola a esta muchacha, porque esta muchacha viene la criatura muerta y tengo que estar yo allí a la fuerza”. Y entonces ya de que acabó con la otra, ya bajó a mi casa pero entonces estaba ya mi hijo en el mundo y yo lavada y todo. (Informante de Mancha Real).

En los pueblos donde las matronas tenían más edad, las parteras eran más dadas a intervenir:

Les decía doña Carmen "anda ve y le recoges a fulana que yo no puedo subir, que ya no puedo". Había muchas cuestas y la mujer estaba mayor y mandaba a Isabel o a Agustina o a cualquiera. (Informante de Cambil).

No obstante eran los médicos los primeros interesados en hacer desaparecer el trabajo de las parteras, les interesaba más el trabajo que hacían las matronas, les daba más seguridad y tranquilidad, ellos, según testimonio de una matrona que ejerció en uno de estas localidades (La Guardia), eran los que más velaban por erradicar el intrusismo profesional. Donde no pudieron desligarse del trabajo de las parteras era en pedanías o municipios muy pequeños. En algunos de ellos parece que no hubo matrona titulada.

\section{DISCUSIÓN}

Las complejas relaciones establecidas por las matronas de esta comarca rural del sur de España, durante la segunda mitad del siglo XX quedan reflejadas en el mapa conceptual que presentamos.

Investigaciones realizadas en otros paises han revisado los temas de formación e institucionalización de las enfermeras con formación de matrona (nurses-midwives) ${ }^{(6,16)}$. La pérdida de poder de las matronas (nurse-midwife) en Estado Unidos guarda una estrecha similitud con la situación vivida en Europa, en ambos continentes estas mujeres se enfrascaron en una lucha con el colectivo de médicos por delimitar las parcelas de actuación. Curiosamente ambos colectivos estuvieron unidos previamente para erradicar la partería u obstetricia tradicional ${ }^{(17)}$.

Nuestro estudio pone en evidencia las características de las relaciones entre las matronas tituladas y las parteras (matronas tradicionales). Fueron unas relaciones de un cierto grado de cooperación inicial, para pasar a continuación a una sustitución progresiva. Las parteras (matronas tradicionales) desempeñaron una función importante en la atención al parto en las comarcas rurales de España, de forma similar a los hallazgos de autores en países culturalmente relacionados, como México $(18,19)$ o de culturas diferentes como Estados Unidos (20) o Sudáfrica (21). 
Los límites al desarrollo profesional de las matronas tituladas se pusieron de manifiesto por la carencia de control sobre sus actuaciones y la subordinación al colectivo de médicos ${ }^{(22)}$. Otras interesantes investigaciones de autoras inglesas han contribuido al estudio de las matronas analizando su trabajo técnico en los siglos XIX y XX, y la autonomía profesional en relación a las nuevas tecnologías, además incorporan el análisis de género a los procesos de cambio, las relaciones con las pacientes, las relaciones entre profesionales y los modos de profesionalización ${ }^{(23)}$.

El estudio de las matronas rurales de esta comarca del sur de España hay que enmarcarlo dentro del discurso sociopolítico del régimen dictatorial que gobernaba el país en el tiempo estudiado, un discurso construido sobre la doble autoridad del patriarcado tradicional y científico con el que se trataba de recubrir la perpetua e interesada tutela sobre las mujeres (24). Este control social de género encontró su paralelismo en las instituciones sanitarias donde los médicos, apoyados por el Estado, sentaron las bases del trabajo complementario pero jerárquico de las matronas, resaltando las limitaciones de la matrona en su práctica y cuándo ésta debía dejar paso al trabajo más importante del médico.

Este estudio que aquí presentamos nos ha permitido comprobar cómo en el entorno rural de Mágina las matronas fueron marcando su territorio y adquiriendo protagonismo. Los médicos dejaron a las matronas la dirección de los partos, de esta forma la jerarquía teórica del sistema sanitario fue invertida en la realidad de la práctica rural. Esta autoridad de las matronas fue reforzada por las mujeres de los pueblos al considerarlas a estas como las profesionales referentes para la asistencia al parto normal. Los practicantes-ATS y parteras tradicionales supieron difuminarse de la esfera del parto cuando las matronas ocuparon sus plazas, unos y otras lo hicieron de forma progresiva sin causar disturbios.

Las matronas rurales creyeron en la toma de sus propias decisiones, desarrollando un sentimiento de orgullo profesional. la división del espacio laboral entre el colectivo médico y de matronas, refleja que la intervención de éstas a través de su experiencia, les dotó de un sentido de independencia profesional. los contenidos formativos que aprendieron en las aulas fueron transformados en función del espacio y contexto donde trabajaron, lo que en la práctica les otorgó autoridad. en consonancia con el estudio que Maxine Rhodes ${ }^{(25)}$ realizó en Gran Bretaña, podemos afirmar que el estudio de las relaciones sociales y laborales que las matronas de Sierra Mágina mantuvieron, nos permite acercarnos a la formación de la identidad profesional que configuraron como colectivo.

Los testimonios orales de los actores sociales y protagonistas ofrecen información completa del pasado ya que la valoración de la experiencia vivida de cada persona permite el estudio de las relaciones sociales de género. El estudio del día a día con vecinos y profesionales de la salud y el estudio del impacto del trabajo de las matronas nos aproxima más a su realidad, a su estatus y a las cotas de poder que alcanzaron. Las condiciones de trabajo y las funciones de la matrona rural, generalizada en otros pueblos de la geografía española, como responsable y referente para la asistencia del nacimiento marcó para este colectivo un estatus social y sanitario que se ha ido desdibujando y perdiendo espacio de poder con la incorporación generalizada de la asistencia obstétrica a los hospitales y maternidades en los años 70 del siglo XX.

\section{REFERENCIAS}

1. Del Val Valdivieso, M.I. (2004). "A modo de introducción. La historia en los albores del siglo XXI". [An introduction. The Histoy in the beginning of the XXIth], pp. 11-27 in: Del Val 
Valdivieso et al. (Eds.), La Historia de las Mujeres: Una revisión Historiográfica. [The women's history. A historiographic review] Universidad de Valladolid, Valladolid.

2. Nash, M. (1996). "Historia e historiografía de las mujeres españolas". [Spanish women's history and historiography], pp. 385-404. in: García de León, M.A. and García de Cortazar, M., Sociología de las mujeres españolas. [Spanish women's sociology]. Editorial Complutense, Madrid.

3. Bernabeu, J. and Cotanda, S. (1991). "Antecedentes históricos del proceso de socialización de la enfermería española". [Historic background of Spanish nursing]. Enfermería Cientifica. 111: 28-36.

4. Blasco Ordoñez, C. (1993). "La incorporación de practicantes y matronas al sistema sanitario español (1901-1950)". [Inclusion of healthcare technicians and midwives to Spanish Health system (1901-1950)]. Index de Enfermería. II (4-5): 7-10.

5. Gómez Ruiz, M.D. (1997). "Matronas: ¿cómo ha evolucionado la profesión?” [Midwives: how the profession developed?]. Revista ROL de Enfermería. XX(221): 71-74.

6. Dawley, K. (2002). "Perspectives on the past, view of the present: relationship between nurse-midwifery and nursing in the United States". Nursing Clinics of North America, 37 (4): 747-755.

7. Ortiz Gómez, T. (2001). "El género organizador de las profesiones sanitarias". [Gender as organizer in health professions], pp. 53-76 in Miqueo, C. et al.(Eds.), Perspectivas de género en salud. Fundamentos científicos y socioprofesionales de diferencias sexuales no previstas. [Gender outlook in health. Scientific and socio-professional basics for no-predicted sexual differences]. Minerva Ediciones, Madrid.

8. Krueger, Richard A. (1991). "El grupo de discusión. Guía práctica para la investigación aplicada". [Discussion group. Practice guide for applied research]. Pirámide, Madrid.

9. García Calvente, M.M. and Mateo Rodríguez, I. (2000). "El grupo focal como técnica de investigación cualitativa en salud: diseño y puesta en práctica". [Discussion group as a qualitative research method: design and implementation]. Atención Primaria, 25, (3): 181186.

10. Karnblit, AL. (2004). "Metodologías cualitativas en ciencias sociales". [Qualitative methods in social sciences]. Bibles, Buenos Aires.

11. Barrio Cantalejo, I. and Simón Lorda P. (2006). "Problemas éticos de la investigación cualitativa" [Ethic issues in qualitative research]. Medicina Clínica, 126 (11): 418-423.

12. Huberman, M. and Miles, M.B. (1994). "Data management and analysis methods", pp. 428-444 in: Denzin N.K. and Lincon, Y.S., (Eds.), Handbook of qualitative research. Thousand Oaks, CA. Sage.

13. López Pegalajar, M. (2002). "Ritos de nacimiento y muerte en Sierra Mágina". [Rites from birth and death in Sierra Magina]. Sumuntán, 17: 95-108.

14. Linares Abad, M. et al. (2002). "La relación de ayuda a la mujer durante el embarazo, parto y puerperio del primer hijo". [The relationship of help to women during first child pregnancy and birth]. Index de Enfermería, 38: 9-14.

15. Bernabeu, J. (2002). "Madres y enfermeras. Demografía y salud en la política poblacionista del primer franquismo, 1939-1950". [Mothers and nurses. Demographic and health in first franquismo population politic, 1939-1950] Revista de demografía histórica XX, I: 123-143.

16. Stone, S.E. (2000). "The evolving scope of nurse-midwifery practice in the United States". Journal Midwifery Women Health, 45(6): 522-531.

17. Scoggin, J. (1997). "The historical relationship of nurse-midwifery with medicine". Journal Nurse Midwifery, 42(1): 47-52.

18. Bortin, S. (1993). "Interviews with Mexican midwives". Journal of Nurse Midwifery. 38(3). 170-177.

19. Trueba, G. (1994). “Midwifery in Mexico”. Modern Midwife, 4(4): 28-29. 
20. Ventre, F. et al. (1995). "The transition from lay mid wife to certified nurse-midwife in the United States". Journal of Nurse Midwifery, 40(5): 428-437.

21. Troskie, T.R. (1997). "The importance of traditional midwives in the delivery of health care in the republic of South Africa". Curationis, 20(1): 15-20.

22. Mcintosh, T. (1998). "Profession, skill, or domestic duty?". Social history of medicine, 11(13): 403-420.

23. Marland, H. and Rafferty, A.M. (Eds.) (1997). "Midwives, society and childbirth. Debates and controversies in the modern period". Routledge. London.

24. Palacio Lis, I. (2003). "Mujeres ignorantes: madres culpables. Adoctrinamiento y divulgación materno-infantil en la primera mitad del siglo XX". [lgnorant women: guilty mothers. Indoctrination and spreading for mothers and children in first half of the XX century]. Universidad de Valencia, Valencia.

25. Rhodes, M. (2001). "Saber y práctica de la matronería en Gran Bretaña, 1936-1950". [Knowledge and practice in Midwifery in United Kingdom, 1936-1950], pp.189-213 in: Cabré, M. and Ortiz. T. Sanadoras, matronas y médicas en Europa. [Healer, midwives and physician women in Europe]. Icaria, Barcelona. 